\title{
Disrupted cell skeletons may explain brain wiring changes in autism-linked condition
}

\author{
BY NIKO MCCARTY
}

28 JUNE 2021

Mutations in TSC2, a gene linked to autism and a related condition called tuberous sclerosis complex, cause developing neurons to ignore chemical cues that help them connect with each other, a new study suggests. The results may explain the altered wiring patterns seen in the brains of people with such mutations.

TSC2 mutations disrupt the formation of axons, the long neuronal projections that send electrical signals from one brain cell to another, past research shows. Researchers long attributed this faulty wiring to problems with mTOR, a signaling pathway that helps neurons synthesize proteins and other materials they need to grow and form connections; without TSC2, mTOR runs amok.

In neurons derived from the skin of a person with tuberous sclerosis, however, the mTOR pathway is not hyperactive, the new study found. Instead, the work implicates a different signaling protein: RhoA.

"We were very surprised," says Timothy Gómez, professor of neuroscience at the University of Wisconsin-Madison, who led the new study. "We were expecting this to be all mTOR."

RhoA helps neurons reshape an internal cytoskeleton as they extend axons toward other cells. Deletions or duplications of genes in the Rho pathway are found in people with autism. Several genes in the autism-linked chromosomal region 16p11.2 also interact with RhoA.

"I'm glad to see that many autism-related genes are now converging on RhoA, which actually makes a lot of sense," says Lilia lakoucheva, associate professor of psychiatry at the University of California, San Diego, who was not involved in the study. "The work is beautiful."

\section{Misguided axons:}




\section{Spectrum | Autism Research News}

https://www.spectrumnews.org

Gómez's team created glutamatergic cortical neurons, which mediate excitatory signals in the brain, using stem cells derived from an 18-year-old man with tuberous sclerosis and autism. The cells carried a TSC2 mutation that results in low levels of TSC2 protein but no changes in mTOR activity relative to control neurons.

The researchers placed these neurons between parallel lines made of ephrin- $A$, a chemical that prevents migrating axons from from going astray and linking up incorrectly in the developing brain.

Axons from cells missing one copy of TSC2 ignored the ephrin-A boundaries, branching out and growing uncontrollably. These axons also stretched farther than those from control neurons, which grew in straight lines, rigidly avoiding the chemical.

Developing axons from neurons missing one copy of TSC2 respond typically to ephrin-A if exposed first to high doses of lysophosphatidic acid (LPA), a RhoA activator, a 2010 study showed. Gómez's team replicated that experiment with their own TSC2 neurons; the results hint that TSC2 mediates axon growth by way of RhoA.

"If you give cells enough LPA, RhoA shoots up, and then axons collapse," Gómez says.

He and his colleagues also engineered some of the TSC2 neurons to produce more RhoA protein, and the developing axons grew as expected: They skirted away from ephrin-A lines and also responded to other guidance cues, confirming RhoA's role in the cell's migration problems. The results appeared 10 May in Nature Communications.

"This is the first study I know of that has shown that an autism disorder creates these guidance defects," Gómez says.

Missed connections: Developing axons with just one functional copy of the TSC2 gene (right) ignore inhibitory cues (red stripes), unlike axons with two copies (left).

Timothy Gómez, University of Wisconsin-Madison

\section{Unknown links:}

RhoA signaling defects are implicated in many autism-related conditions. But researchers should be cautious in extrapolating the new results to those other conditions, says Mustafa Sahin, director of the Translational Neuroscience Center at Boston Children's Hospital in Massachusetts. Sahin led the 2010 work and peer-reviewed the new study prior to publication. 


\section{Spectrum | Autism Research News}

https://www.spectrumnews.org

"Different genes can regulate the cytoskeleton and Rho activity in different ways," he says. "In most of our work on tuberous sclerosis, loss of TSC in one neural type versus another has very different effects."

For instance, 16p11.2 deletion in neurons that release the neurotransmitter dopamine causes an increase in RhoA activity, according to a May study by Sahin's team — the opposite of what Gómez's team found.

And problems with mTOR and protein synthesis are clearly not the whole story for tuberous sclerosis, either. "Most of us focus on protein synthesis, but we also think there are differences in the transcription of genes and there are differences in the cytoskeleton, through RhoA," Sahin says.

Everolimus, a medication that inhibits mTOR, is approved by the U.S. Food and Drug Administration for treating seizures associated with tuberous sclerosis. Ongoing clinical trials are also testing rapamycin, an mTOR inhibitor, for treating benign tumors caused by the condition. Now, "future treatments may have to take into account all these different things that TSC regulates," Sahin says, including RhoA.

Gómez and his colleagues studied dynamic axon projections rather than neurons alone, says Karun Singh, associate professor of medicine the University of Toronto in Canada, who was not involved in the study. Other researchers should do the same, he says, because axon growth and its role in forming neuronal connections during development is understudied in autism research.

Gómez, whose father, Manuel Rodríguez Gómez, edited the first textbook on tuberous sclerosis and established diagnostic criteria for the condition, says he intends to continue this work. His group plans to study the biochemical signals that directly link TSC2 to RhoA, using the same axon growth model.

"This is the most exciting work with human stem cells to come out of my lab," he says. "We're really hopeful that we can make more contributions."

Cite this article: https://doi.org/10.53053/LYED2893 